\title{
A QoS-Based Robust Multipath Routing Protocol for Mobile Adhoc Networks
}

\author{
S.Venkatasubramanian and Dr.N.P.Gopalan
}

\begin{abstract}
In Mobile ad hoc networks, the unstable transport layer and inhibited amount of traffic being carried out by the network is owing to the high packet loss rates and frequent topological changes. It is essential that least available bandwidth and end-to-end latency along with congestion around a link are integrated in a QoS-based routing metric for MANETs. In this paper we develop a QOS-based, Robust Multipath Routing (QRMR) protocol for mobile ad hoc networks to allot weights to individual links, depending on the metrics link quality, channel quality and end-to-end delay. The individual link weights are combined into a routing metric to validate the load balancing and interference between links using the same channel. Consequently, the traffic is balanced and the network capacity is improved as the weight value assists the routing protocol to evade routing traffic through congested area. Subsequently, the selection of the proportion of traffic to be routed to each neighbor is made to perform routing such that the weight of the node is a possible minimum. We illustrate the robustness of our protocol as it accomplishes increased packet delivery ratio with reduced latency, through simulation results.
\end{abstract}

Index Terms-Bandwidth, MANETs, Multipath, Packet Loss, QoS, Routing Protocol, QRMR.

\section{INTRODUCTION}

\section{A. Quality of Service (QoS) in MANET}

Ad hoc wireless network is a special case of wireless network devoid of predetermined backbone infrastructure. This feature of the wireless ad hoc networks makes it flexible and quickly deployable. Nevertheless, significant technological challenges are also posed by this property. There are several challenges incorporating issues of efficient routing, medium access, power management, security and quality of service (QoS). As the nodes correspond over wireless links, all the nodes must combat against the extremely erratic character of wireless channels and intrusion from the additional transmitting nodes. These factors make it a challenging problem to exploit on data throughput even if the user-required QoS in wireless ad hoc networks is achieved.

Repeated route changes cause huge complications in implementing ad hoc networks owing to the mobility of the nodes and intrusion between nodes. The high packet loss

S.Venkatasubramanian is now with Saranathan college of Engineering, Trichy, 620012, India. Phone No. +91 9443144356; fax: 91-431-2473684; India. rates and recurrent topological changes lead to unbalanced transport layer and constrained amount of traffic being carried out by the network.

The three eminent problems in ad hoc networks are the lack of unswerving packet delivery due to the intrusion and movement of nodes, incomplete bandwidth owing to the channel limitations, and constrained node life span caused as an outcome of small battery size.

\section{B. Problems of Adhoc Routing Protocols}

(i) The massive dimension of ad hoc networks necessitates an excellent scalable QoS architecture. An ad hoc network may not want (or be able) to limit the number of hosts involved in the network. As more nodes join an ad hoc network or the data traffic grows, the potential for collisions and contention increases, and protocols face the challenging task to route data packets and result in scalability. The prior works on QoS in ad hoc networks have barely considered the scalability problems.

(ii) The packets which are equipped to be transmitted are buffered by the Network Interface Queues (IFQ) implemented by AODV [1], TORA [2], and DSR [3] and the network protocol stack receives these packets. In general, the utmost numbers of packets that can be held in the IFQ are limited and in addition a maximum timeout policy for packets in the IFQ is implemented by IFQ. Hence, devoid of any notification, any packet in anticipation of a route in the IFQ for an extensive period may well merely be discarded [4]. Hou and Tipper [5] state that the brim over of the IFQ of congested nodes is one of the key motives for the decline in throughput for congested networks running DSR.

(iii) The communication Gray Zone problem is an important challenge encountered by the existing routing protocols [4]. The detection and establishment of end to end routes are performed in most of the routing protocols such as AODV by depending on the control (RREQ) packets. Nevertheless, these control packets and data packets have appreciably different properties. For instance, In comparison to data packets, RREQ packets are in general greatly smaller and are also transmitted at considerably lower bit rates. Nodes that are distant might be able to transmit and receive bidirectional RREQ packets, however high bit-rate data packets cannot be sent or received as a consequence of the above mentioned property. High control overhead for protocols such as AODV is acquired due to the consequent highly "fragile" link that triggers link repairs.

(iv) End-to-end delay estimation is a vital element of any QoS-enabled routing protocol. Current protocols determine 
the time taken to route RREQ and RREP packets along the specified path in order to estimate end-to-end delay. Nevertheless, RREQ and RREP packets are improbable to encounter the similar levels of traffic delay and loss like data packets as they are appreciably diverse from usual data packets.

We depend on the reality that communication is based on received power of the forwarding node, in order to be able to observe the movement of the node and that a route is about to break.

As a result, the quality of the route is measured. The link breakage can be guessed based on the measured quality of the route. By using a proactive fault tolerant routing with QoS aware multipath route discovery, smaller end-to-end delay and a larger throughput to a host can be achieved.

In this paper, we develop a QOS-based, Robust Multipath Routing (QRMR) protocol intended for mobile ad hoc networks. In this paper, we will employ the facility to determine multiple routes to a host and switch between them to expand the definition of AOMDV [6].Enabling a QoS constrained route from source to destination is one of the objectives of the routing protocol. The route chosen by the protocol must send packets with minimum bandwidth and end-to-end latency, in particular. The protocol should satisfy the above constraints and also select the most robust among all possible candidate routes.

The remainder of the paper is organized as follows. Section 2 reviews some of the existing work in this area. Section 3 presents the system design and overview. Section 4 presents the details of estimating the QoS based routing metrics. Section 5 describes our proposed QoS based robust multipath routing protocol in detail. The experimental results are presented in Section 6 and conclude the paper in Section 7.

\section{RELATED WORK}

Andre Schumacher et al. [7] have approached the problem of load balancing for wireless multihop networks by distributed optimization. They have used an approximation algorithm for minimizing the maximum network congestion and implement it as a modification of the DSR routing protocol. The algorithm was based on shortest-path computations that are integrated into the DSR route discovery and maintenance process. Therefore, it does not rely on the dissemination of global information within the entire network.

Kaixin $\mathrm{Xu}$ et al. [8] have proposed a Scalable QoS architecture suitable for large scale mobile ad hoc networks. Important contribution of the proposed architecture was its scalability. Most of the works was pushed to the source nodes. Intermediate nodes only need to perform limited work without the need of any state information. With the help of the scalable LANMAR routing protocol, the proposed QoS scheme works efficiently in large scale ad hoc networks with thousands of nodes. They have also introduced the mobile backbone network structure to enhance the network performance.

Jianbo Xue et al. [9] have proposed a QoS framework for MANETs Adaptive Reservation and Pre-allocation Protocol (ASAP). By using two signaling messages, ASAP provides fast and efficient QoS support while maintaining adaptation flexibility and minimizing wasted reservations.

Jangeun Jun and Mihail L. Sichitiu [10] have exposed a significant fairness problem existent practically in all wireless multihop networks. They have also proposed several network layer solutions to the fairness problem. The improvement in fairness was directly related to the resources investment. They showed that a network layer solution can restore fairness at the expense of bandwidth efficiency. In addition they have also shown that a MAC layer providing priorities was able to restore network efficiency while maintaining network layer fairness. Finally, they have generalized the fairness concept to enable differentiated services in multihop networks.

Michael Gerharz et al. [11] have taken a practical view on the Quality-of-Service capabilities of wireless ad hoc networking technologies. They have outlined that it is a very complex task to reserve or even measure available bandwidth in wireless multihop networks. They have introduced and evaluated the DLite algorithm, an approach to service differentiation in ad hoc networks. It applies a fair queuing scheme with separate queues for each service class. Late packets of delay constrained classes were dropped in intermediate routers. DLite allows for adaptive multimedia applications and permits gradual deployment.

Duc A. Tran and Harish Raghavendra [12] have proposed CRP a congestion-adaptive routing protocol for MANETs. CRP enjoys fewer packet losses than routing protocols that are not adaptive to congestion. This is because CRP tries to prevent congestion from occurring in the first place, rather than dealing with it reactively. A key in CRP design was the bypass concept. Because a bypass is removed when the congestion is totally resolved, CRP does not incur heavy overhead due to maintaining bypass paths. The bypass maintenance cost was further reduced because a bypass is typically short and a primary node can only create at most one bypass.

Xiaoqin Chen et al. [13] have proposed a congestionaware routing protocol for mobile ad hoc networks which uses a metric incorporating data-rate, MAC overhead, and buffer delay to combat congestion. This metric was used, together with the avoidance of mismatched link data-rate routes, to make mobile ad hoc networks robust and adaptive to congestion.

Ming Yu et al. [14] have proposed a link availability-based QoS-aware (LABQ) routing protocol for mobile ad hoc networks based on mobility prediction and link quality measurement, in addition to energy consumption estimate. Their goal was to provide highly reliable and better communication links with energy-efficiency. Their routing algorithm has been verified by NS-2 simulations and the results have shown that LABQ outperforms existing algorithms by significantly reducing link breakages and 
thereby reducing the overheads in reconnection and retransmission. It also reduces the average end-to-end delay for data transfer and enhances the lifetime of nodes by making energy-efficient routing decisions

\section{System Design AND Protocol OVERVIEW}

We devise the model by considering a mobile ad hoc network in which each node utilizes IEEE 802.11 DCF for medium access control (MAC). At the application layer, intra-flow interference occurs for the same flow which is transmitted on different wireless links. But it has been presumed as a single data flow.

We put forward a QOS based multipath routing protocol intended for mobile ad hoc networks. In this paper, we will employ the facility to determine multiple routes to a host and switch between them to expand the definition of AOMDV [6].

Enabling a QoS constrained route from source to destination is the objective of the routing protocol. The route chosen by the protocol must send packets with minimum bandwidth and end-to-end latency, without facing congestion. The protocol should satisfy the above constraints and also select the most robust among all possible candidate routes.

A QoS-based routing metric for MANETs should incorporate minimum available bandwidth and end-to-end latency along with congestion around a link. Congestion is related to channel quality, which depends on the MAC access contention and channel reliability. So our algorithm should rely on the following metrics to allocate weights to individual links.

- $\quad$ End-to-End Delay

- Channel Quality

- Link Quality

The individual link weights are combined into a routing metric to validate the load balancing and interference between links using the same channel. Consequently, the traffic is balanced and the network capacity is improved as the weight value assists the routing protocol to evade routing traffic through congested area. Subsequently, the selection of the proportion of traffic to be routed to each neighbor is made to perform routing such that the weight of the node is a possible minimum.

\section{Estimating QoS-Based Routing Metrics}

\section{A. Estimating Link Quality}

Each node in the network estimates its quality of links with its one-hop neighbors. If $N q$ is the number of HELLO packets received during a time window Twin and $P q$ is the percentage of HELLO packets received in the last $r$ seconds, then the link quality $L q$ is measured as

$$
L q=\delta \cdot \mathrm{Pq}+(1-\delta) \cdot \mathrm{Nq}
$$

The estimated link quality is maintained by each node in its NT. The average link quality of all the links across the path $P$, gives the route quality $R q$ of the path. RREQ packets of the reverse path and RREP packets of the forward path accumulate the estimated $L q$ values.

\section{B. Estimating End to End Delay}

There is a significant variation between the end-to-end delay reported by RREQ-RREP measurements and the delay experienced by actual data packets. We address this issue by introducing a DUMMY-RREP phase during route discovery. The source saves the RREP packets it receives in a RREP TABLE and then acquires the RREP for a route from this table to send a stream of DUMMY data packets along the path traversed by this RREP. DUMMY packets efficiently imitate real data packets on a particular path owing to the same size, priority and data rate as real data packets. $\mathrm{H}$ is the hop count reported by the RREP. The number of packets comprised in every stream is $2 \mathrm{H}$. The destination computes the average delay $D_{a v g}$ of all DUMMY packets received, which is sent through a RREP to the source. The source selects this route and sends data packets only when the average delay reported by this RREP is inside the bound requested by the application. The source performs a linear back-off and sends the DUMMY stream on a different route selected from its RREP TABLE when the delay exceeds the required limit.

\section{Estimating Channel Quality}

The channel quality can be represented by both the channel occupancy and channel reliability.

\section{1) Channel Occupation}

2) In this network, we consider IEEE 802.11 MAC with the distributed coordination function (DCF). It has the packet sequence as request-to-send (RTS), clear-to-send (CTS), data and acknowledgement (ACK). The amount of time between the receipt of one packet and the transmission of the next is called a short inter frame space (SIFS). Then the channel occupation due to MAC contention will be

$$
C_{o c c}=t_{R T S}+t_{C T S}+3 t_{S I F S}+t_{a c c}
$$

Where $t_{R T S}$ and $t_{C T S}$ are the time consumed on $R T S$ and $C T S$, respectively and $t_{S I F S}$ is the SIFS period. $t_{a c c}$ is the time taken due to access contention.

The channel occupation is mainly dependent upon the medium access contention, and the number of packet collisions. That is, $C_{o c c}$ is strongly related to the congestion around a given node.

$C_{o c c}$ can become relatively large if congestion is incurred and not controlled, and it can dramatically decrease the capacity of a congested link. 
Therefore, in the design of a congestion-aware metric for networks, the access contention should be considered to more accurately indicate channel capacity.

3) Channel Reliability

4) The channel reliability also affects the packet transmission in MANETs and factors such as interference and fading in the channel may lead to occurrence of packet losses. In 802.11 DCF, several failed retransmissions lead to packets being dropped. In addition to performance deterioration due to congestion with respect to packet losses, higher packet retransmissions involved owing to augmented packet losses lead to more congestion. A successful transmission of a packet on an unreliable links would consume more time on MAC overhead.

\section{QoS Based Robust Multipath Routing Protocol}

For establishing multiple disjoint paths, we adapt the idea from the Adhoc On-Demand Multipath Distance Vector Routing (AOMDV) [6]. The multiple paths are computed during the route discovery.

We now introduce the weight metric $\mathrm{W}$ which assigns a cost to each link in the network. The weight $\mathrm{W}$ combines the link quality $L q$, channel quality $C_{o c c}$ and the average delay $D_{a v g}$, to select maximum throughput paths, avoiding the most congested links.

For an intermediate node $i$ with established transmission with several of its neighbours, the $W$ for the link from node $i$ to a particular neighboring node is given by

$$
W=L q+C_{o c c}+D_{a v g}
$$

\section{A. Route Request}

During the route discovery phase of the protocol, each intermediate node uses an admission control scheme to check whether the flow can be accepted or not. If accepted, a Flow Table (FT) entry for that particular flow is created. The FT contains the fields Source (Src), Destination (Dst), Reserved Bandwidth (BWres), Minimum bandwidth (BWmin). Each node collects the bandwidth reserved at its one hop neighbors (piggybacked on periodic HELLO packets) and stores it in its Neighbor Table (NT) .The Neighbor Table contains fields Destination (Dst), Reserved Bandwidth (BWres), No. of Hello Packets (No Hello).

Consider the scenario

Let us consider the route

$$
W=L q+C_{o c c}+D_{a v g}
$$

To initiate QoS-aware routing discovery, the source host $S$ sends a $R R E Q$. When the intermediate host $R 1$ receives the RREQ packet, it first estimates all the metrics as described in the previous section.

The host $R 1$ then calculates its weight $W_{R I}$ using (3).

$$
R R E Q_{R I} \stackrel{W_{R 1}}{\longrightarrow} R 2
$$

$R 2$ then calculates its weight $W_{R 2}$ in the same way and adds it to the weight of $R 1$. $R 2$ then forward the $R R E Q$ packet with this added weight.

$$
R R E Q_{R 2} \stackrel{W_{R 1}+W_{R 2}}{\longrightarrow} R 3
$$

Finally the $R R E Q$ reaches the destination node $D$ with the sum of node weights

$$
R R E Q_{R 3} \stackrel{W_{R 1}+W_{R 2}+W_{R 3} \longrightarrow D}{\longrightarrow} D
$$

\section{B. Route Reply}

The Destination node $D$ send the route reply packet $R R E P$ along with the total node weight to the immediate upstream node $R 3$.

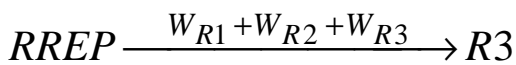

Now $R 3$ calculates its cost $C$ based on the information from $R R E P$ as

$$
C_{R 3}=\left(W_{R 1}+W_{R 2}+W_{R 3}\right)-\left(W_{R 1}+W_{R 2}\right)
$$

By proceeding in the same way, all the intermediate hosts calculate its cost.

On receiving the $R R E P$ from all the routes, the source selects the route with minimum cost value.

We will show that these calculations will not increase the overhead significantly, by simulation results in the next section.

\section{SimULATION RESULTS}

\section{A. . Simulation Model and Parameters}

We use NS2 to simulate our proposed protocol. In our simulation, the channel capacity of mobile hosts is set to the same value: 2 Mbps. We use the distributed coordination function (DCF) of IEEE 802.11 for wireless LANs as the MAC layer protocol. It has the functionality to notify the network layer about link breakage.

In our simulation, mobile nodes of sizes 25, 50, 75 and 100 move in a 1000 meter x 1000 meter rectangular region for 100 seconds simulation time. We assume each node moves independently with the same average speed. All nodes have the same transmission range of 250 meters. In our simulation, the minimal speed is $5 \mathrm{~m} / \mathrm{s}$ and maximal speed is $20 \mathrm{~m} / \mathrm{s}$. The simulated traffic is Constant Bit Rate (CBR).

Our simulation settings and parameters are summarized in table 1

TABLE I

SimUlation PARAMETERS

\begin{tabular}{|l|l|}
\hline No. of Nodes & $25,50,75$ and 100 \\
\hline Area Size & 1000 X 1000 \\
\hline Mac & 802.11 \\
\hline Radio Range & $250 \mathrm{~m}$ \\
\hline $\begin{array}{l}\text { Simulation } \\
\text { Time }\end{array}$ & $100 \mathrm{sec}$ \\
\hline
\end{tabular}




\begin{tabular}{|l|l|}
\hline Traffic Source & CBR \\
\hline Packet Size & 512 \\
\hline Mobility Model & $\begin{array}{l}\text { Random Way } \\
\text { Point }\end{array}$ \\
\hline Speed & $5 \mathrm{~m} / \mathrm{s}$ to $20 \mathrm{~m} / \mathrm{s}$ \\
\hline Pause time & $5 \mathrm{~s}$ \\
\hline
\end{tabular}

\section{B. Performance Metrics}

We compare our QRMR protocol with the AOMDV [6] protocol. We evaluate mainly the performance according to the following metrics, by varying the nodes as $25,50,75$ and 100.

Control overhead: The control overhead is defined as the total number of routing control packets normalized by the total number of received data packets.

Average end-to-end delay: The end-to-end-delay is averaged over all surviving data packets from the sources to the destinations.

Average Packet Delivery Ratio: It is the ratio of the No. of packets received successfully and the total no. of packets sent

\section{Results}

\section{Varying No. Of Nodes}

In the first experiment, we measure the performance of the protocols by varying the no. of nodes as 25, 50, 75 and 100 .

From Fig. 1, we can see that the packet delivery ratio for QRMR increases, when compared to AOMDV, since it utilizes robust links.

From Fig. 2, we can see that the average end-to-end delay of the proposed QRMR protocol is less when compared to the AOMDV protocol.

Fig. 3 shows that the control overhead of the protocols. Since QRMR make use of HELLO packets for cost estimation, the values are considerably less in QRMR when compared with AOMDV.

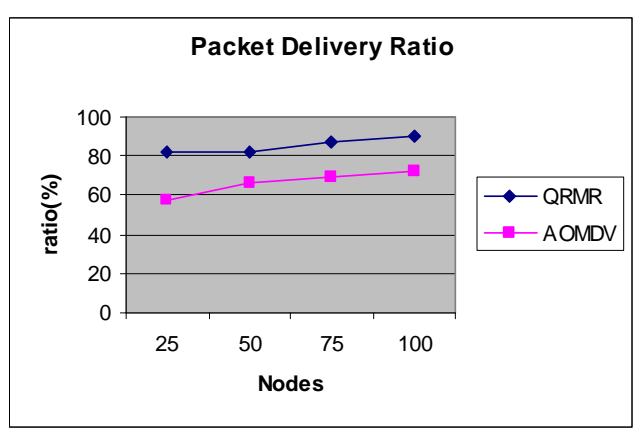

Fig. 1 Nodes Vs Delivery Ratio

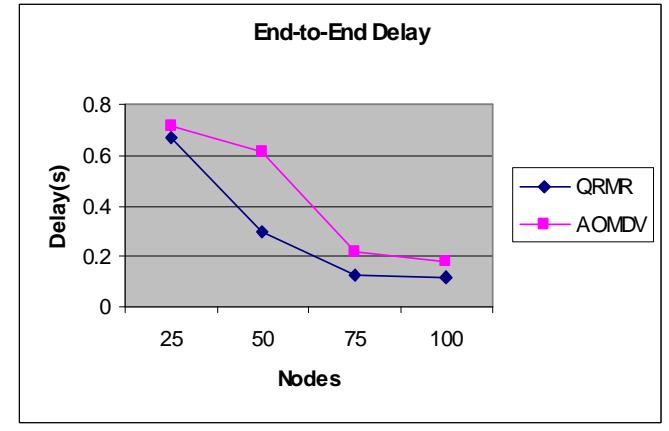

Fig. 2 Nodes Vs Delay

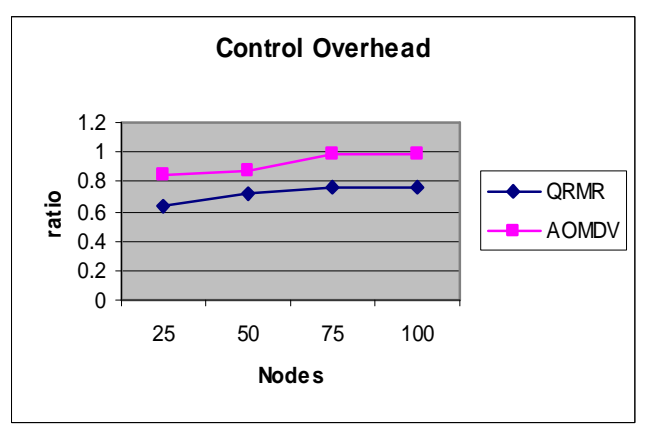

Fig. 3 Nodes Vs Overhead

\section{Varying the Transmission Rate}

In the second experiment, we measure the performance of the protocols by varying the transmission rate as $250,500,750$ and $1000 \mathrm{~Kb}$.

From Fig. 4, we can see that the packet delivery ratio for QRMR is more, when compared to AOMDV, since it utilizes robust links.

From Fig. 5, we can see that the average end-to-end delay of the proposed QRMR protocol is less when compared to AOMDV.

Fig. 6 shows the control overhead of the protocols. Since QRMR make use of HELLO packets for cost estimation, the values are considerably less in QRMR when compared with AOMDV.

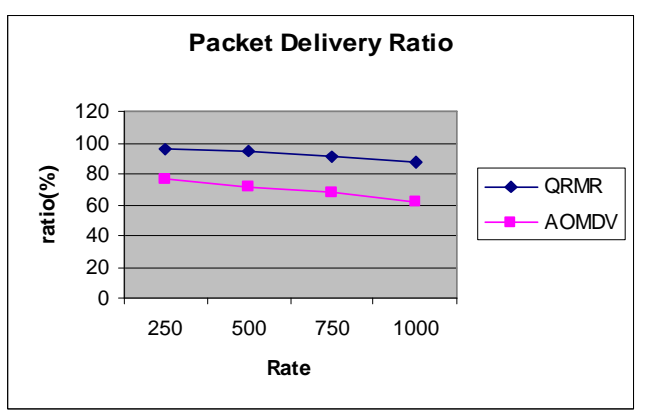

Fig. 4 Rate Vs Delivery Ratio

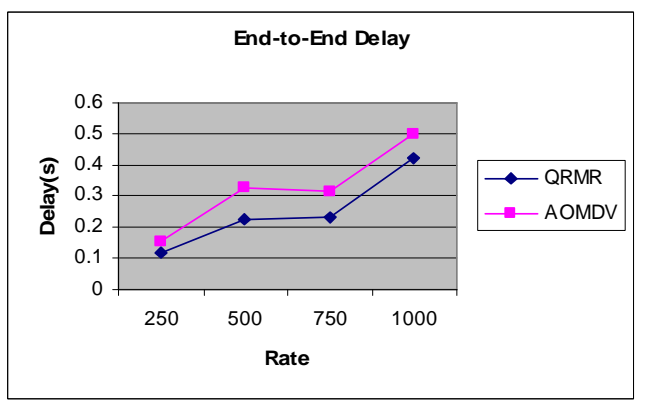


Fig.5 Rate Vs Delay

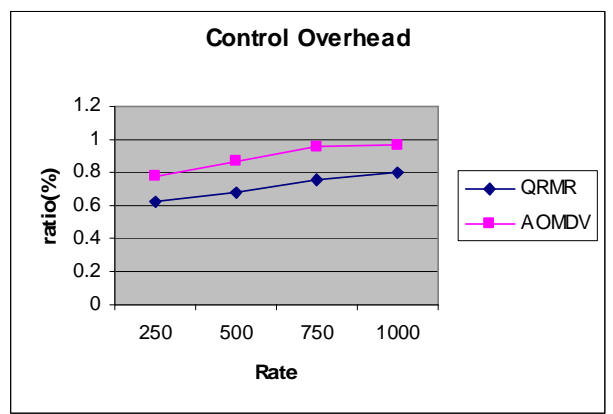

Fig. 6 Rate Vs Overhead

\section{CONCLUSION}

It is essential that least available bandwidth and end-to-end latency along with congestion around a link are integrated in a QoS-based routing metric for MANETs. MAC access contention and channel reliability influences congestion which is related to channel quality. In this paper, we have devised a QOS-based, Robust Multipath Routing (QRMR) protocol intended for mobile ad hoc networks. It allocates weights to individual links on the basis of the metrics link quality, channel quality and end-to-end delay. The traffic is balanced and the network capacity is improved as the weight value assists the routing protocol to evade routing traffic through congested area. Subsequently, the selection of the proportion of traffic to be routed to each neighbor is made to perform routing such that the node weight is a possible minimum. We have illustrated the robustness of our protocol as it accomplishes inc packet delivery ratio with reduced latency, through simulation results.

The security challenges of our proposed protocol will be the subject of our future work. Also the future work involves comparison of our proposed protocol with some more exiting protocols.

\section{REFERENCES}

[1] Charles E. Perkins and Elizabeth M. Royer, "Ad-hoc On-Demand Distance Vector Routing", in proceedings of 2nd workshop on Mobile Computing Systems and Applications, 25-26 Feb., New Orleans, LA, USA, 1999, pp: 90- 100.

[2] Vincent D. Park and M. Scott Corson, "A Highly Adaptive Distributed Routing Algorithm for Mobile Wireless Networks", in Proc. of IEEE INFOCOM'97, vol. 3,7-12 April, Kobe, Japan, 1997, pp: 1405-1413.

[3] David B. Johnson and David A. Maltz, "Dynamic Source Routing in Ad Hoc Wireless Networks", in proceedings of International Series in Engineering and Computer Science, vol. 353,1996, pp: 153- 181.

[4] Asad Amir Pirzada and Chris McDonald, "Inherent Robustness of Reactive Routing Protocols against Selfish Attacks", in proceedings of International Workshop on Wireless Ad-hoc Networks, London, England, 2005.

[5] Xiaobing Hou and David Tipper, "Impact of Failures on Routing in Mobile Ad Hoc Networks Using DSR", in Web Protocols White Papers, University of Pittsburgh, November 2002.

[6] Mahesh K. Marina and Samir R. Das, "On- Demand Multipath Distance Vector Routing in Ad Hoc Networks", in proceedings of 9th IEEE International Conference on Network Protocols, 11- 14 November 2001, pp: 14- 23.

[7] Andre Schumacher, Harri Haanpaa, Satu Elisa Schaeffer, and Pekka Orponen, "Load Balancing by Distributed Optimization in Ad Hoc
Networks", in proceedings of 2nd International Conference on Mobile Ad-hoc and Sensor Networks, vol. 4325,Berlin- Heidelberg 2006, pp: 874- 885 .

[8] Kaixin Xu, Ken Tang, Rajive Bagrodia, Mario Gerla and Michael Bereschinsky, "Adaptive Bandwidth Management and QoS Provisioning in Large Scale Ad Hoc Networks", in proceedings of IEEE Conference on Military Communications, vol. 2,13- 16, 2003,pp: 1018- 1023.

[9] Jianbo Xue, Patrick Stuedi and Gustavo Alonso, "ASAP: An Adaptive QoS Protocol for Mobile Ad Hoc Networks", IEEE Proceedings on Personal, Indoor and Mobile Radio Communications, vol. 3,7- 10 September 2003, pp: 2616- 2620.

[10] Jangeun Jun and Mihail L. Sichitiu, "Fairness and QoS in Multihop Wireless Networks", in proceedings of 58th IEEE Conference on Vehicular Technology, vol. 5, 6- 9 October 2003, pp: 2936- 2940.

[11] Michael Gerharz, Christian de Waal, Matthias Frank and Paul James, "A Practical View on Quality-of-Service Support in Wireless Ad Hoc Networks", Proc. of the 3rd IEEE Workshop on Applications and Services in Wireless Networks (ASWN), Berne, Switzerland, July 2003, pp: 185-196.

[12] Duc A. Tran and Harish Raghavendra, "Congestion Adaptive Routing in Mobile Ad Hoc Networks", IEEE Transactions on Parallel and Distributed Systems, Vol. 17, no. 11, November 2006.

[13] Xiaoqin Chen, Haley M. Jones, A .D .S. Jayalath, "Congestion-Aware Routing Protocol for Mobile Ad Hoc Networks", in proceedings of 6th IEEE Conference on Vehicular Technology,30 September- 3 October, Baltimore, 2007, pp: 21- 25.

[14] Ming Yu , Aniket Malvankar, Wei Su , Simon Y. Foo , "A link availability-based QoS-aware routing protocol for mobile ad hoc sensor networks", Journal on Computer Communications, vol. 30, no. 18, December 2007, pp: 3823-3831.

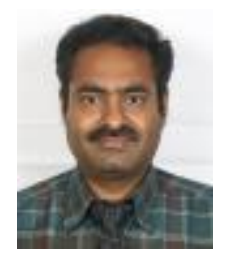

S.Venkatasubramanian received the B.E. degree in Electronics and Communication from Bharathidasan University and M.E. degree in Computer science from Regional Engineering College. $\mathrm{He}$ has 13 years of teaching experience. He is currently pursuing doctoral research in mobile Ad hoc networks. His areas of interest include mobile networks, Network Security and software engineering. At present he is working as Assistant Professor in Department of CSE at Saranathan college of Engineering, Trichy, India

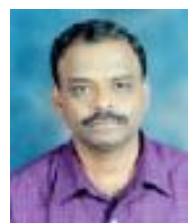

Dr.N.P.Gopalan is a Professor in the Department of computer application at National Institute of Technology, Tiruchirapalli. He received the M.E. degree in Computer science from Regional Engineering College. and Ph.D degree in theoretical computing from Indian Institute of Science .He has more than 30 years of teaching experience. He has published more than 20 papers in International and National journals and conference proceedings. He has published 3 books. His areas of research include Data mining, Web Technology, Distributed Computing, and Theoretical Computer Science. 